\title{
A new approach for noninvasive transdermal determination of blood uric acid levels
}

\author{
This article was published in the following Dove Press journal: \\ International Journal of Nanomedicine \\ 28 June 2014 \\ Number of times this article has been viewed
}

\author{
Congo Tak-Shing Ching ${ }^{1,2}$ \\ Kok-Khun Yong ${ }^{3}$ \\ Yan-Dong Yao ${ }^{4}$ \\ Huan-Ting Shen ${ }^{3}$ \\ Shiu-Man Hsieh ${ }^{5}$ \\ Deng-Yun Jheng' \\ Tai-Ping Sun ${ }^{1,6}$ \\ Hsiu-Li Shieh'
}

'Department of Electrical Engineering, National Chi Nan University, Nantou, ${ }^{2}$ Department of Photonics and Communication Engineering, Asia University, Taichung, ${ }^{3}$ Department of Internal Medicine, Puli Christian Hospital, Nantou, Taiwan, Republic of China; ${ }^{4}$ Division of Science and Technology, Hong Kong Community College, Hong Kong; ${ }^{5}$ Department of Orthopedic Surgery, Puli Christian Hospital, ${ }^{6}$ Department of Electronic Engineering, Nan Kai University of Technology, Nantou, Taiwan, Republic of China
Correspondence:

Congo Tak-Shing Ching

Department of Electrical Engineering, National Chi Nan University,

I University Rd, Puli, Nantou County

5456I, Taiwan, Republic of China

Tel +88 64 929l 0960 ext 4774

Email tsching@ncnu.edu.tw

Kok-Khun Yong

Puli Christian Hospital, Teh-Shan Rd,

Puli, Nantou County 54546, Taiwan,

Republic of China

Email khunIII0@mail.pch.org.tw

\begin{abstract}
The aims of this study were to investigate the most effective combination of physical forces from laser, electroporation, and reverse iontophoresis for noninvasive transdermal extraction of uric acid, and to develop a highly sensitive uric acid biosensor (UAB) for quantifying the uric acid extracted. It is believed that the combination of these physical forces has additional benefits for extraction of molecules other than uric acid from human skin. A diffusion cell with porcine skin was used to investigate the most effective combination of these physical forces. UABs coated with $\mathrm{ZnO}_{2}$ nanoparticles and constructed in an array configuration were developed in this study. The results showed that a combination of laser $(0.7 \mathrm{~W})$, electroporation $\left(100 \mathrm{~V} / \mathrm{cm}^{2}\right)$, and reverse iontophoresis $\left(0.5 \mathrm{~mA} / \mathrm{cm}^{2}\right)$ was the most effective and significantly enhanced transdermal extraction of uric acid. A custom-designed $\mathrm{UAB}$ coated with $\mathrm{ZnO}$, nanoparticles and constructed in a $1 \times 3$ array configuration $\left(\mathrm{UAB}-1 \times 3-\mathrm{ZnO}_{2}\right)$ demonstrated enough sensitivity $(9.4 \mu \mathrm{A} / \mathrm{mM})$ for quantifying uric acid extracted by the combined physical forces of laser, electroporation, and RI. A good linear relationship $\left(R^{2}=0.894\right)$ was demonstrated to exist between the concentration of uric acid $(0.2-0.8 \mathrm{mM})$ inside the diffusion cell and the current response of the UAB- $1 \times 3-\mathrm{ZnO}_{2}$. In conclusion, a new approach to noninvasive transdermal extraction and quantification of uric acid has been established.
\end{abstract}

Keywords: laser, electroporation, reverse iontophoresis, noninvasive, uric acid, biosensor

\section{Introduction}

Gout is a common form of arthritis ${ }^{1}$ with many sufferers, comprising about 3 million people in the USA ${ }^{2}$ and 0.7 million in the UK. ${ }^{3}$ Moreover, gout accounts for 4 million outpatient visits each year, ${ }^{4}$ resulting in a considerable economic burden. ${ }^{5}$ Recent research shows a link between gout or high blood uric acid levels and increased risk of cardiovascular morbidity and mortality. ${ }^{6}$ Therefore, frequent monitoring of blood uric acid levels is of great importance.

Blood sampling is a routine clinical approach to determining blood uric acid levels. However, it suffers the drawback of being invasive, painful, and inconvenient. To offer patients a noninvasive alternative, physical forces such as laser, ${ }^{7,8}$ reverse iontophoresis (RI), ${ }^{9-14}$ and electroporation ${ }^{13-15}$ have been investigated both independently and in combination for their ability to extract metabolites noninvasively and transdermally.

Laser perforation is a technique whereby micropores are created in the skin by pulsed laser to promote transport of molecules. ${ }^{7,8}$ RI refers to the technique of passing a small current through a section of skin to promote transport of both charged and neutral molecules. ${ }^{16}$ Electroporation refers to the process of creating transient micropores across cell membranes by injecting a brief high voltage pulse to permit movement of exogenous molecules. ${ }^{17}$ Several papers have reported the use of 
combined physical forces to enhance transdermal extraction of metabolites. ${ }^{13,14,18,19}$ To the best of our knowledge, no research has been reported on the use of combined physical forces from laser, RI, and electroporation to extract uric acid noninvasively and transdermally. Therefore, laser, electroporation, and RI were used in this study to evaluate the effectiveness of these physical forces with regard to noninvasive transdermal extraction of uric acid.

Uric acid extracted transdermally can generally be quantified using analytical tools such as a colorimeter or biosensor. Amongst the analytical tools available, a biosensor is the best choice for convenient quantification of uric acid at home. Due to the usually low concentration of uric acid that can be extracted transdermally, biosensors for this purpose should ideally have high sensitivity. Many researchers have focused on the use of nanoparticles, ${ }^{20,21}$ carbon nanotubes, ${ }^{22,23}$ electrontransfer mediators, ${ }^{24,25}$ bimetallic materials, ${ }^{26,27}$ bienzymes, ${ }^{22,23}$ and array configurations ${ }^{28}$ to enhance the sensitivity of biosensors. To the best of our knowledge, there have been no reports of the use of a combination of the above approaches to enhance biosensor sensitivity. Hence, this study investigates the use of integrated nanoparticles and an array configuration for improving the sensitivity of biosensors.

The aims of this study were to: (1) identify the most effective combination of physical forces (from laser, electroporation, and RI) for noninvasive transdermal extraction of uric acid; (2) develop a highly sensitive uric acid biosensor (UAB) for quantifying the uric acid extracted by physical forces; and (3) integrate the extraction method in (1) with the highly sensitive UAB in (2) for development of a system to determine blood uric acid levels by transdermal and noninvasive means.

\section{Materials and methods Reagents and solutions}

All reagents were commercially available and used without further purification in this study. Uricase, uric acid, bovine serum albumin, glutaraldehyde, phosphate-buffered saline, and $\mathrm{ZnO}_{2}$ nanoparticles were purchased from Sigma Chemical Co (St Louis, MO, USA). A K608-100 uric acid assay kit was sourced from BioVision (Mountain View, CA, USA). Deionized water (resistivity $\geq 18 \mathrm{~m} \Omega \mathrm{cm}$ ) purified by a Millipore system (Milli-Q UFplus; Bedford, MA, USA) was used to prepare all solutions. A Screen-print sensor (three electrode configuration, ie, graphite working and counter electrodes, and a silver-silver chloride reference electrode from Zensor R\&D Co Ltd, Taichung, Taiwan).

\section{Equipment}

A previously described diffusion cell (Figure 1) ${ }^{13}$ was used for the in vitro uric acid extraction studies, with components including: programmable iontophoresis ${ }^{29}$ and electroporation devices $^{30}$ (developed by the authors to provide the necessary physical forces for electroporation and RI); a modified commercial laser generation device $(1,550 \mathrm{~nm}$ laser modules, Tang Yue Ltd, Taiwan) to provide another essential physical force; a 680 microplate reader (Bio-Rad, Hemel Hempstead, UK) for all colorimetric analyses; and a CHI 627C electrochemical analyzer ( $\mathrm{CH}$ Instruments, Austin, TX, USA) for all electrochemical evaluations.

\section{In vitro uric acid extraction studies}

All uric acid extraction experiments were conducted using diffusion cells, having two upper electrode chambers (300 $\mu \mathrm{L}$ each) and one lower chamber $(25 \mathrm{~mL})$, separated by $250 \mu \mathrm{m}$-thick porcine ear skin (obtained by dermatome) to simulate human skin. All chambers were filled with $0.1 \mathrm{M}$ phosphate-buffered saline ( $\mathrm{pH}$ 7.0), and the lower chamber contained additional $400 \mu \mathrm{M}$ uric acid to simulate the normal interstitial fluid under human skin.

The experimental protocols are summarized in Figure 2, in which three types of physical forces, ie, laser, electroporation, and RI, were investigated for their effect on transdermal extraction of uric acid. In the experiments requiring application of electroporation, a pair of platinum (Pt) electrodes was positioned in the electrode chambers and the required electroporation was delivered by the programmable electroporation device via the Pt electrodes. In the experiments requiring application of RI, a pair of silver-silver chloride $(\mathrm{Ag} / \mathrm{AgCl})$ electrodes was positioned

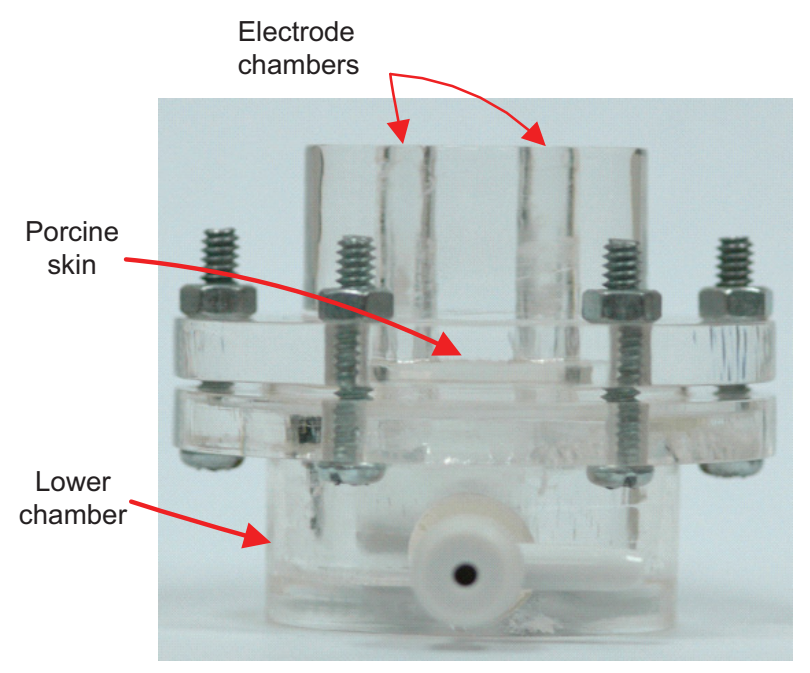

Figure I Diffusion cell used for in vitro uric acid extraction studies. 
A

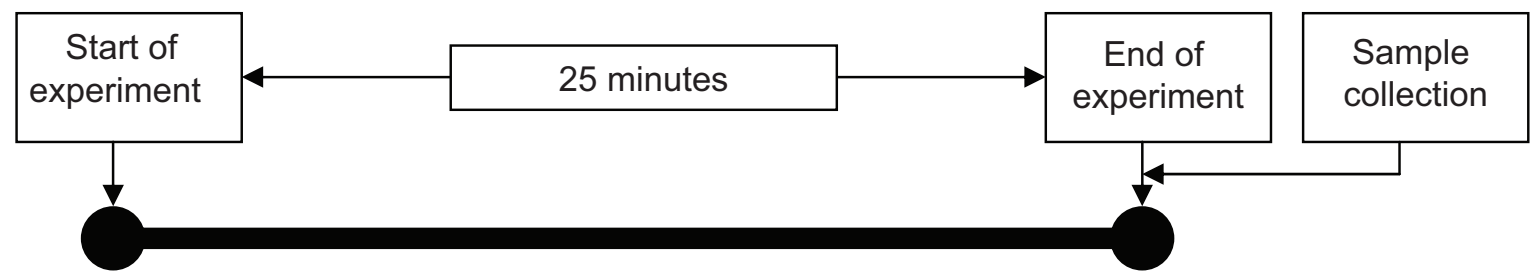

B

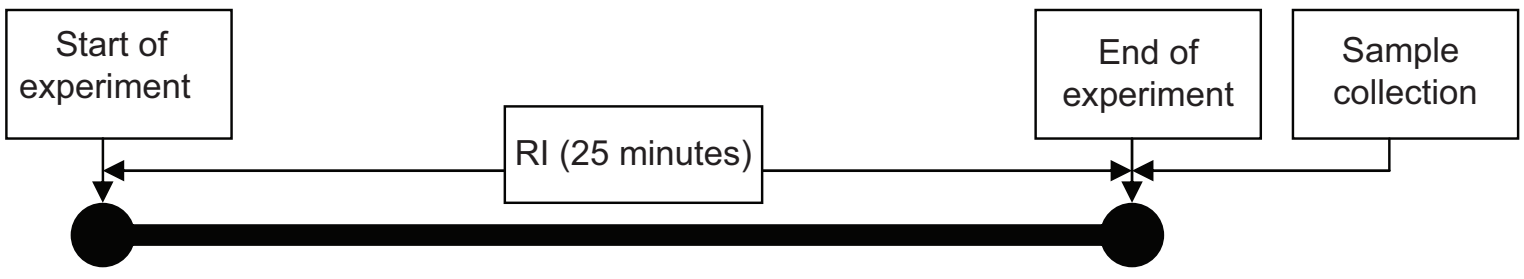

C

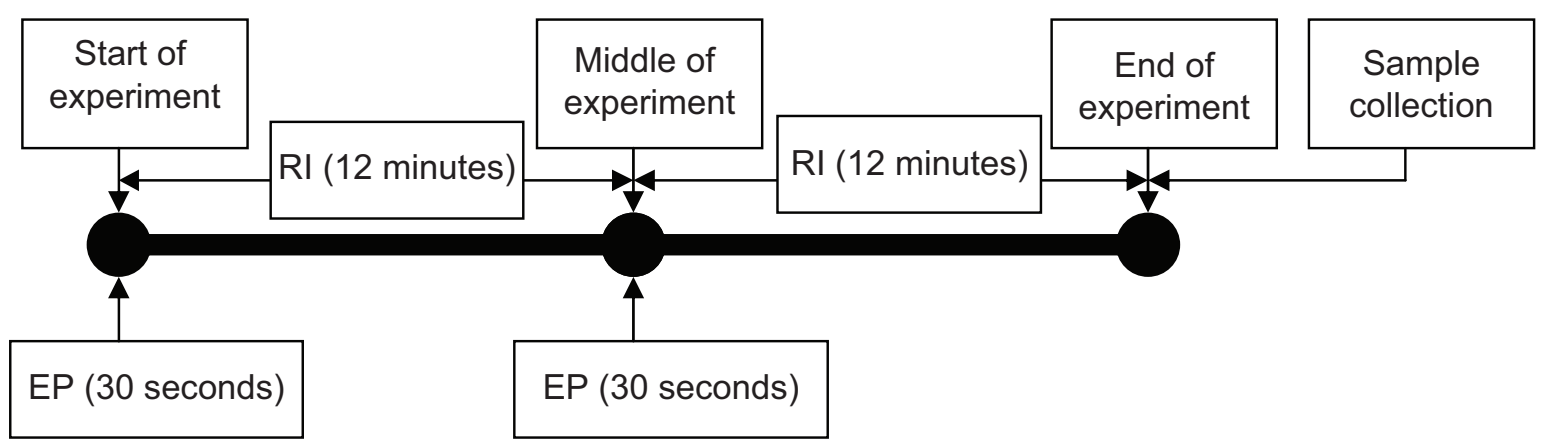

D
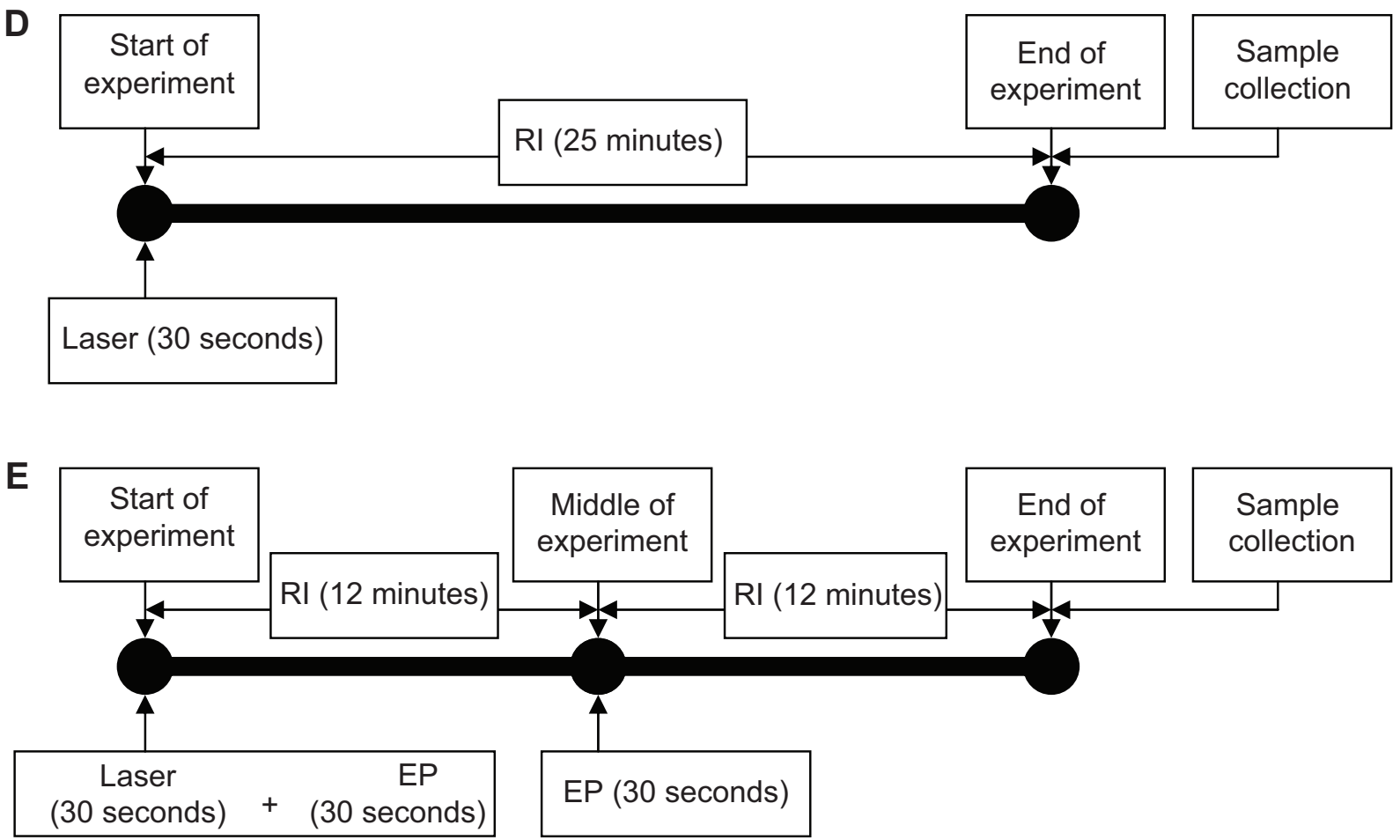

Figure 2 The experimental protocols.

Notes: Each protocol allows 25 minutes of extraction time. For RI, symmetrical biphasic DC (phase duration I 80 seconds; current density $0.5 \mathrm{~mA} / \mathrm{cm}^{2}$ ) was used. For EP, high electric pulses (pulse width I msec; 20 pulses per second; polarity reversing every 5 seconds; application time 30 seconds; voltage 50 or 100 V/ $\mathrm{cm}^{2}$ ) were used. For laser, a near-infrared laser (wavelength I,500 nm; pulse width 10 msec; duty cycle 50\%; application time 30 seconds; power $0.3 \mathrm{~W}$ or $0.7 \mathrm{~W}$ ) was used. (A) control group (ie, no $\mathrm{RI}$, EP, and laser used), (B) application of RI alone, (C) application of EP and RI, (D) application of laser and RI, and (E) application of laser, EP, and RI.

Abbreviations: EP, electroporation; RI, reverse iontophoresis; DC, direct current. 
in the electrode chambers and the required RI was delivered by the programmable iontophoresis device via the $\mathrm{Ag} /$ $\mathrm{AgCl}$ electrodes. Both the $\mathrm{Pt}$ and $\mathrm{Ag} / \mathrm{AgCl}$ electrodes were positioned $1 \mathrm{~mm}$ above the porcine skin in their respective experiments. In experiments requiring application of laser, the porcine skin was treated by laser directly before the two electrode chambers were filled up with $0.1 \mathrm{M}$ phosphatebuffered saline ( $\mathrm{pH} 7.0)$.

At the end of each extraction experiment, the entire contents of the electrode chambers were removed to determine the quantity of uric acid extracted by colorimetric assay, using the uric acid assay kit and Bio-Rad microplate reader.

\section{Construction of $U A B$ array incorporated with $\mathrm{ZnO}_{2}$ nanoparticles}

A commercially available three-electrode sensor was used in this study. To immobilize the uricase enzyme on the sensor, a $4 \mu \mathrm{L}$ mixture of glutaraldehyde $2.5 \%$ and $\mathrm{ZnO}_{2}$ nanoparticles (20\% weight/weight [w/w]) was initially drop-coated onto the working electrode, and the sensor was then kept at $4{ }^{\circ} \mathrm{C}$ in dark for one hour. Subsequently, $4 \mu \mathrm{L}$ of uricase $(0.5 \mathrm{U} / \mathrm{mL})$ was drop-coated again onto the working electrode, immediately followed by drop-coating of $4 \mu \mathrm{L}$ of bovine serum albumin $(0.1 \mathrm{mM})$. Finally, the sensor was again kept at $4^{\circ} \mathrm{C}$ in the dark before use. The same procedure was used to construct $\mathrm{UAB}$ without $\mathrm{ZnO}_{2}$ nanoparticles but with $4 \mu \mathrm{L}$ of glutaraldehyde $2.5 \%$ alone.

In this study, four configurations of UAB (Figure 3 ) were investigated in order to determine the sensitivity of biosensors with different configurations and nanoparticle coatings. The configurations were: single UAB (UAB- $1 \times 1$, Figure $3 \mathrm{~A}$ ), single $\mathrm{UAB}$ with $\mathrm{ZnO}_{2}$ nanoparticles $\left(\mathrm{UAB}-1 \times 1-\mathrm{ZnO}_{2}\right.$,
Figure $3 \mathrm{~A}$ ), UAB with $\mathrm{ZnO}_{2}$ nanoparticles in a $1 \times 2$ array configuration $\left(\mathrm{UAB}-1 \times 2-\mathrm{ZnO}_{2}\right)($ Figure $3 \mathrm{~B})$, and UAB with $\mathrm{ZnO}_{2}$ nanoparticles in a $1 \times 3$ array configuration (UAB- $1 \times 3$ $\mathrm{ZnO}_{2}$, Figure 3C).

The procedures for $\mathrm{UAB}-1 \times 1$ and $\mathrm{UAB}-1 \times 1-\mathrm{ZnO}_{2}$ construction are the same. To construct UAB- $1 \times 2-\mathrm{ZnO}_{2}$, two pieces of $\mathrm{UAB}-1 \times 1-\mathrm{ZnO}_{2}$ were positioned in parallel. Similarly, UAB- $1 \times 3-\mathrm{ZnO}_{2}$ was constructed by positioning three pieces of $\mathrm{UAB}-1 \times 1-\mathrm{ZnO}_{2}$ in parallel.

\section{Measurements of current responses of $U A B$ to uric acid}

Measurement of the rate of hydrogen peroxide $\left(\mathrm{H}_{2} \mathrm{O}_{2}\right)$ generation during enzymatic oxidation of uric acid is the basis of the mechanism for $\mathrm{UAB}$ detection, where $\mathrm{H}_{2} \mathrm{O}_{2}$ is electroactive and $\mathrm{UAB}$ employs amperometric transduction. ${ }^{31}$ The current signal of UAB corresponds to the increase in exposure to uric acid solution. The oxidation of uric acid $\left(\mathrm{C}_{5} \mathrm{H}_{4} \mathrm{~N}_{4} \mathrm{O}_{3}\right)$ to allantoin $\left(\mathrm{C}_{4} \mathrm{H}_{6} \mathrm{~N}_{4} \mathrm{O}_{3}\right)$ by uricase in the presence of oxygen $\left(\mathrm{O}_{2}\right)$ is shown below:

$$
\mathrm{C}_{5} \mathrm{H}_{4} \mathrm{~N}_{4} \mathrm{O}_{3}+\mathrm{O}_{2}+2 \mathrm{H}_{2} \mathrm{O} \stackrel{\text { Uricase }}{\longrightarrow} \mathrm{C}_{4} \mathrm{H}_{6} \mathrm{~N}_{4} \mathrm{O}_{3}+\mathrm{CO}_{2}+\mathrm{H}_{2} \mathrm{O}_{2} \text {. }
$$

The current response of the $\mathrm{UAB}$ to uric acid was evaluated by an electrochemical analyzer at $25^{\circ} \mathrm{C}$. A voltage of $+700 \mathrm{mV}$ versus the $\mathrm{Ag} / \mathrm{AgCl}$ electrode was provided to the working electrode of the UAB.

To measure the current response, phosphate-buffered saline was first pipetted onto the UAB and left for 3 minutes to allow stabilization of the background current, before uric acid solutions of various concentrations were pipetted onto the $\mathrm{UAB}$ and the current response recorded. The data were then used to plot a UAB current response-uric acid
A

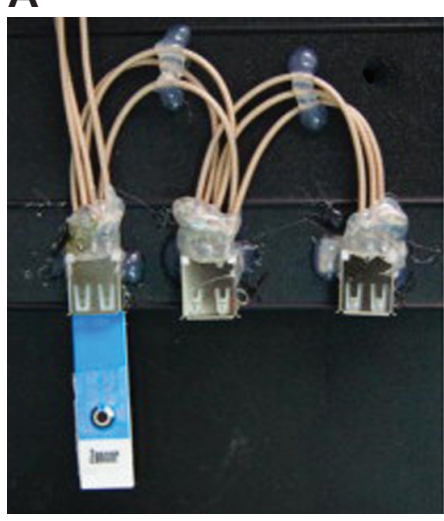

B

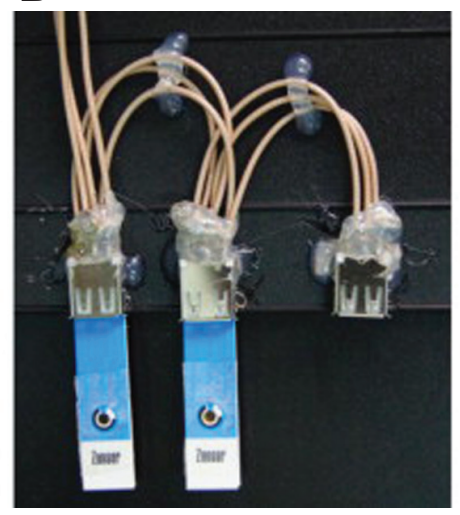

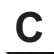

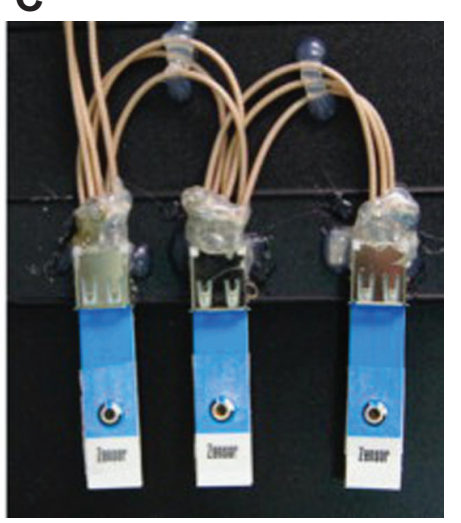

Figure 3 (A) A single UAB. UAB-I $\times I$ denotes $U A B$ without a $\mathrm{ZnO}_{2}$ nanoparticle coating, while $U A B-I \times I-Z n O_{2}$ denotes one with a $\mathrm{ZnO}{ }_{2}$ nanoparticle coating. (B) $A$ UAB in $\mathrm{I} \times 2$ array configuration. When the $\mathrm{ZnO}_{2}$ nanoparticle coating was deployed, it is abbreviated to $\cup A B-I \times 2-\mathrm{ZnO}$, and constructed by fixing two $\cup A B-I \times I-Z n O_{2}$ in parallel. (C) $A \cup A B$ in $I \times 3$ array configuration. With a $\mathrm{ZnO}_{2}$ nanoparticle coating on, it is abbreviated to $U A B-I \times 3-Z n O_{2}$, and constructed by fixing three $U A B-I \times I-Z n O_{2}$ in parallel. Note: Copyright (C) 2013, Elsevier B.V. Reproduced from Ching CTS, Chang KM, Hung YL, et al. An array configuration to increase the performance of a biosensor. Sens Actuators B Chem. 2013; 178:465-472. ${ }^{28}$

Abbreviation: UAB, uric acid biosensor. 
concentration curve, from which the sensitivity of $U A B$ was determined as the slope of a linear regression line fitted to the data.

\section{Statistical analysis}

Independent-samples $t$-test was also used to determine whether there are significant differences between $U A B$ in different configurations on its sensitivity. A linear regression test was used to determine the relationship between UAB current responses and the standard uric acid solution at different concentrations, as well as the uric acid concentration in diffusion cells. All statistical analyses were carried out using Statistical Package for the Social Sciences software (SPSS Inc., Chicago, IL, USA), with the level of statistical significance set at $P<0.05$.

\section{Results}

Significant enhancement of transdermal extraction of uric acid was found with the use of physical forces $(P<0.05)$ as compared with diffusion alone (Figure 4), a combination of physical forces was found to enhance (significant in most cases with $P<0.05$ ) a higher transdermal extraction of uric acid as compared to RI alone. When comparing electroporation combined with RI and laser combined with RI, a significant boost of transdermal extraction was also apparent for the former $(P<0.05$, in most cases), which is similar to the relationship between the use of three combined physical forces and two physical forces ( $P<0.05$, in most cases).

A highly linear relationship $\left(R^{2} \geq 0.970\right)$ was found to exist between the UAB current response and the uric acid concentration (Figure 5). Addition of $\mathrm{ZnO}_{2}$ nanoparticles to UAB was found to significantly increase $(P<0.05)$ its sensitivity, which was further significantly $(P<0.05$ in all cases) increased by constructing the UAB in array configurations.

A close linear relationship $\left(R^{2}=0.894\right)$ was evident between the concentration of uric acid inside the diffusion cell and the current responses of $\mathrm{UAB}-1 \times 3-\mathrm{ZnO}_{2}$ (Figure 6).

\section{Discussion}

\section{In vitro uric acid extraction studies}

Noninvasive transdermal extraction of uric acid by different physical forces, ie, electroporation, RI, and laser alone

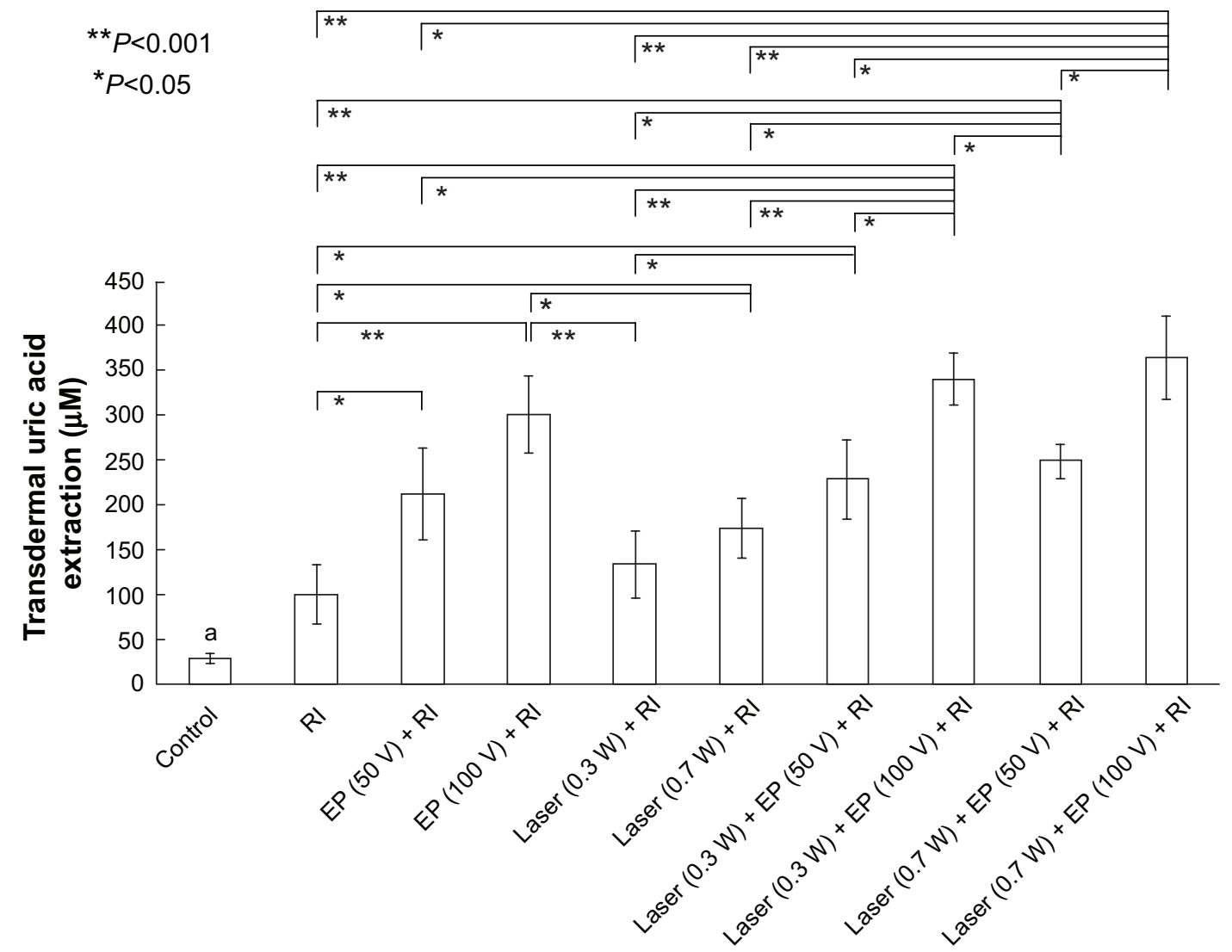

Figure $4 \mathrm{In}$ vitro studies of transdermal noninvasive extraction of uric acid by different physical forces. Statistically significant differences $(* P<0.05$ and $* * P<0.00 \mathrm{I})$ were found. Data are expressed as the mean and standard deviation $(n=3)$.

Note: aUric acid extraction in the control group was found to be significantly $(P<0.05)$ lower than in all other groups.

Abbreviations: EP, electroporation; RI, reverse iontophoresis. 


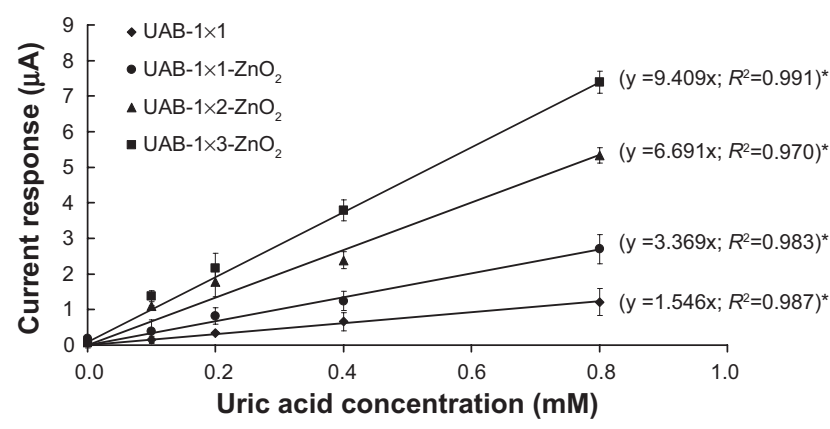

Figure 5 Calibration curves for $U A B$ to uric acid concentration $(0-0.8 \mathrm{mM})$. The lines represent best fit found by linear regression. The sensitivity of $U A B$ is indicated by the slope of the linear regression line.

Notes: * Statistically significant $(P<0.05)$ differences were found for sensitivity when compared with the other UABs. Data are expressed as the mean and standard deviation $(n=3)$.

Abbreviation: $\cup A B$, uric acid biosensor.

or in combination are shown in Figure 4. It was found that application of physical forces can significantly $(P<0.05)$ facilitate transdermal extraction of uric acid as compared with diffusion alone (Figure 2A), ie, a control group without application of physical forces.

Combination of physical forces, such as electroporation combined with RI (Figure 2C), laser combined with RI (Figure 2D), and laser combined with electroporation and RI (Figure 2E), was shown to enhance (significantly in most cases, with $P<0.05$ ) transdermal extraction of uric acid as compared with RI alone (Figure 2B). This is thought to be due to electroporation and the ability of the laser to create nanochannels in the skin, ${ }^{7,8,13-15}$ which further facilitate extraction of uric acid as compared with RI alone. The higher the electroporation voltage (or laser power), the greater the

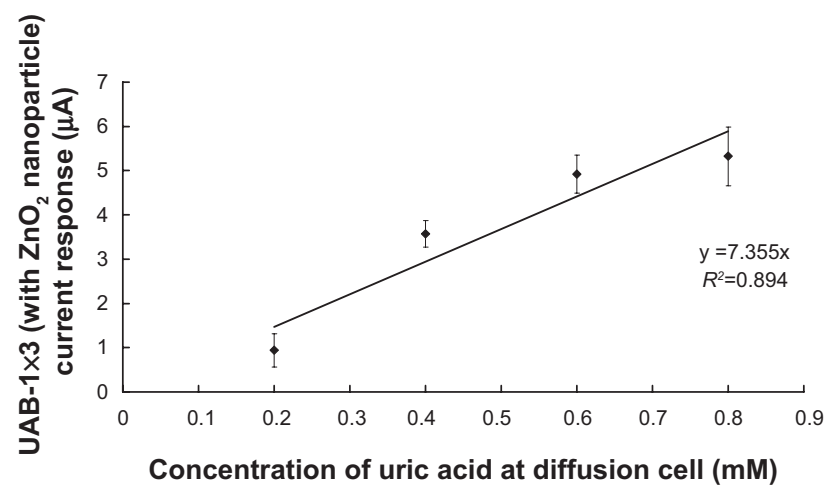

Figure 6 Evaluation of a new approach for noninvasive transdermal determination of uric acid levels.

Notes: A diffusion cell was used, with different concentrations of uric acid $(0.2-0.8 \mathrm{mM})$ stored in its lower chamber. After application of the most effective combination of physical forces, ie, laser $(0.7 \mathrm{~W})+\mathrm{EP}(100 \mathrm{~V})+\mathrm{RI}$ to the porcine skin of the diffusion cell, the concentrations of the extracted uric acid solutions at the upper electrode chambers of the diffusion cell were quantified by $U A B-I \times 3-\mathrm{ZnO}_{2}$. A good linear relationship $\left(R^{2}=0.894\right)$ between the current response of $U A B-1 \times 3-\mathrm{ZnO}_{2}$ and uric acid concentration in the diffusion cell was found. Data are expressed as the mean and standard deviation $(n=5)$.

Abbreviations: EP, electroporation; RI, reverse iontophoresis; UAB, uric acid biosensor. extraction of uric acid. This could be a result of higher electroporation voltage (or laser power) injecting more energy for more formation of nanochannels.

When electroporation is combined with RI (Figure 2C), transdermal extraction of uric acid can be further enhanced $(P<0.05$ in most cases) compared with a combination of laser and RI (Figure 2D). This observation implicates electroporation as a more dominant factor for extraction of uric acid as compared with laser.

Transdermal extraction of uric acid was significantly higher in quantity $(P<0.05$ in most cases) when a combination of three physical forces (ie, laser combined with electroporation and RI, Figure 2E) was used than when two physical forces were used, ie, electroporation combined with RI (Figure 2C) or laser combined with RI (Figure 2D).

In general, the relationship between transdermal extraction of uric acid and physical forces from laser, electroporation, and RI can be seen as follows:

- in order of degree of enhancement of transdermal extraction of uric acid, combination of three physical forces (laser + electroporation $+\mathrm{RI}$ ) $>>$ combination of two physical forces (electroporation + RI or laser + RI; in general, electroporation $+\mathrm{RI}>$ laser $+\mathrm{RI})>>$ single physical force $>>$ diffusion alone

- the higher the electroporation voltage (or laser power), the higher the uric acid extraction.

Based on the above two general relationships and the findings shown in Figure 4, the most effective configurations of laser, electroporation, and RI for uric acid extraction found in this study could be summarized as follows (Figure 2E):

- laser - near-infrared laser; wavelength 1,500 nm; pulse width $10 \mathrm{msec}$; duty cycle $50 \%$; application time 30 seconds; power $0.7 \mathrm{~W}$

- electroporation - high electric pulses; pulse width $1 \mathrm{msec}$; 20 pulses per second; polarity reversing every 5 seconds; application time 30 seconds; voltage $100 \mathrm{~V} / \mathrm{cm}^{2}$

- RI - symmetrical biphasic DC; phase duration 180 seconds; application time 12 minutes; current density $0.5 \mathrm{~mA} / \mathrm{cm}^{2}$.

\section{Uric acid biosensor}

The current responses of the UAB to uric acid concentration $(0-0.8 \mathrm{mM})$ are shown in Figure 5 and show direct proportionality to each other, with a correlation coefficient $\left(R^{2}\right)$ of $\geq 0.970$. Moreover, addition of $\mathrm{ZnO}_{2}$ nanoparticles to the $\mathrm{UAB}$ can significantly increase (2.2-fold, $P<0.05$ ) its sensitivity from $1.546 \mu \mathrm{A} / \mathrm{mM}$ (UAB-1 $1 \times 1$ ) to $3.369 \mu \mathrm{A} / \mathrm{mM}\left(\mathrm{UAB}-1 \times 1-\mathrm{ZnO}_{2}\right)$. The $\mathrm{UAB}$ measurement range used for uric acid in this study was comparable with 
that reported for other sensors, ${ }^{28,32,33}$ but slightly lower than that reported by Zhang et al $(0.2-1 \mathrm{mM}){ }^{34}$ The sensitivity of $\mathrm{UAB}-1 \times 1-\mathrm{ZnO}_{2}(3.369 \mu \mathrm{A} / \mathrm{mM})$ in this study was lower than that reported by Luo et al $(16.6 \mu \mathrm{A} / \mathrm{mM}),{ }^{32}$ but higher than those reported by Zhang et al $(5.5 \mathrm{nA} / \mathrm{mM})^{34}$ and Ching et al $(1.55 \mu \mathrm{A} / \mathrm{mM}){ }^{28}$

The sensitivity of UAB can be significantly increased by the array configuration of UAB. Our results point to UAB- $1 \times 3-\mathrm{ZnO}_{2}$ having a significantly higher $(P<0.05)$ sensitivity when compared with $\mathrm{UAB}-1 \times 1-\mathrm{ZnO}_{2}$ (2.8-fold) and UAB- $1 \times 2-\mathrm{ZnO}_{2}$ (1.4-fold). Further, UAB- $1 \times 2-\mathrm{ZnO}_{2}$ has a significantly $(P<0.05)$ higher sensitivity when compared with $\mathrm{UAB}-1 \times 1-\mathrm{ZnO}_{2}$ (2.0-fold). A possible explanation for these phenomena is that the improved sensitivity is due to the higher signal-to-noise ratio achieved with the larger reductive current signals observed at the UAB in a higher array configuration.

Based on the above, UAB- $1 \times 3-\mathrm{ZnO}_{2}$ was determined to be the most sensitive biosensor for quantification of uric acid extracted transdermally in this study.

\section{Noninvasive and transdermal determination of uric acid levels}

Based on these findings, combining three physical forces was the most effective tool for noninvasive transdermal extraction of uric acid, and the uric acid extracted was best quantified by $\mathrm{UAB}-1 \times 3-\mathrm{ZnO}_{2}$, when diffusion cells as controls for evaluation of this new approach. In this study, uric acid concentrations of $0.2 \mathrm{mM}, 0.4 \mathrm{mM}, 0.6 \mathrm{mM}$, and $0.8 \mathrm{mM}$ was separately placed into the lower chamber of the diffusion cell to simulate low, medium (ie, normal), medium-high, and high patient blood uric acid levels, respectively.

The magnitudes of the current response from UAB$1 \times 3-\mathrm{ZnO}_{2}$ plotted against uric acid concentration inside the diffusion cell is shown in Figure 6, where a well-fitted linear relationship $\left(R^{2}=0.894\right)$ clearly exists between the concentration of uric acid $(0.2-0.8 \mathrm{mM})$ inside the diffusion cell and the current responses from UAB- $1 \times 3-\mathrm{ZnO}_{2}$. This finding demonstrates the accuracy of this new approach to determining uric acid levels noninvasively and transdermally.

\section{Potential applications and limitations of this study}

This study forms the basis of a new approach to noninvasive transdermal determination of blood uric acid levels, which may be relevant to measurements of other metabolites and use of biomarkers. We have validated the claim that biomarkers with a large molecular size, such as prostate-specific antigen ${ }^{13}$ and osteopontin, ${ }^{13}$ can be extracted noninvasively and transdermally by application of combined physical forces of electroporation and RI. However, very high electroporation is required to extract prostate-specific antigen and osteopontin transdermally, which renders this approach clinically impractical. In the present study, a new approach of replacing high electroporation with combined physical forces of low electroporation and low-power laser (Figure 4) makes transdermal extraction of large molecules possible and relatively more effective. As shown in Figure 4, extraction of uric acid by the combined physical forces of low-power laser $(0.3 \mathrm{~W})$, low electroporation $(50 \mathrm{~V})$, and RI, although slightly smaller than that of high electroporation $(100 \mathrm{~V})$ and RI, is comparable. Further study is required to be able to extract large biomarkers successfully and transdermally, for this approach to be clinically practical, when combined physical forces from low-power laser, low electroporation, and RI can be achieved.

However, we have previously demonstrated the possibility of extracting homocysteine noninvasively and transdermally by RI alone, ${ }^{9}$ although the quantity of homocysteine extracted was very low (about $10 \mu \mathrm{M}$ ). Therefore, a highly sensitive biosensor is necessary for detecting such low homocysteine concentrations. In this study, the sensitivity of such biosensors was improved by application of nanoparticles and setting of array configurations, making noninvasive transdermal determination of blood homocysteine levels clinically feasible.

One of the limitations of this study was the application of laser with a wavelength of $1,500 \mathrm{~nm}$, which falls in the invisible spectrum, increasing the chances of eye injury due to accidents. On the other hand, users with cardiac pacemaker may not be able to enjoy the benefit as electroporation may interfere with the function of pacemakers.

\section{Conclusion}

In the present study, a new approach to extracting and quantifying blood uric acid levels noninvasively and transdermally was developed, whereby combinations of physical forces from laser, electroporation, and RI were investigated for noninvasive and transdermal extraction of uric acid, and a highly sensitive $\mathrm{UAB}-1 \times-\mathrm{ZnO}_{2}$ has been developed being capable of accurately quantifying the extracted uric acid.

\section{Acknowledgments}

This work was supported in part by grants from the Ministry of Science and Technology, Taiwan, Republic of China (1022221-E-260-004- and 101-2221-E-260-001-), the Taichung Veterans General Hospital and National Chi Nan University, 
Taiwan, Republic of China (TCVGH-NCNU1027901), and the National Chi Nan University and Puli Christian Hospital, Taichung, Taiwan, Republic of China (102A038).

\section{Disclosure}

The authors report no conflicts of interest in this work.

\section{References}

1. Choi HK, Mount DB, Reginato AM. Pathogenesis of gout. Ann Intern Med. 2005;143:499-516.

2. Lawrence RC, Felson DT, Helmick CG. Estimates of the prevalence of arthritis and other rheumatic conditions in the United States. Arthritis Rheum. 2008;58:26-35.

3. Mikuls TR, Farrar JT, Bilker WB, Fernandes S, Schumacher HR, Saag KG. Gout epidemiology: results from the UK General Practice Research Database, 1990-1999. Ann Rheum Dis. 2005;64:267-272.

4. Krishnam E, Lienesch D, Kwoh CK. Gout in ambulatory care setting in the United States. J Rheumatol. 2008;35:498-501.

5. Wu EQ, Patel PA, Yu AP. Disease-related and all-cause health care costs of elderly patients with gout. J Manag Care Pharm. 2008;14: 164-175.

6. Edwards NL. The role of hyperuricemia and gout in kidney and cardiovascular disease. Cleve Clin J Med. 2008;75 Suppl 5:S13-S16.

7. Service FJ, O’Brien PC, Wise SD, Ness S, LeBlanc SM. Dermal interstitial glucose as an indicator of ambient glycemia. Diabetes Care. 1997;20:1426-1429.

8. Gebhart S, Faupel M, Fowler R, et al. Glucose sensing in transdermal body fluid collected under continuous vacuum pressure via micropores in the stratum corneum. Diabetes Technol Ther. 2003;5:159-166.

9. Ching CTS, Chou TR, Sun TP, Huang SY, Shieh HL. Simultaneous, noninvasive, and transdermal extraction of urea and homocysteine by reverse iontophoresis. Int J Nanomedicine. 2011;6:417-423.

10. Ching CTS, Buisson Y, Connolly P. The effect of pulsed bipolar DC current waveform on the simultaneous extraction of glucose and lactate by reverse iontophoresis. Sens Actuators B Chem. 2008;129: 504-509.

11. Ching CTS, Connolly P. Reverse iontophoresis: a non-invasive technique for measuring blood lactate level. Sens Actuators B Chem. 2008; 129:352-358.

12. Ching TS, Connolly P. Simultaneous transdermal extraction of glucose and lactate from human subjects by reverse iontophoresis. Int J Nanomedicine. 2008;3:211-223.

13. Ching CTS, Fu LS, Sun TP, Hsu TH, Chang KM. Use of electroporation and reverse iontophoresis for extraction of transdermal multibiomarkers. Int J Nanomedicine. 2012;7:885-894.

14. Lee CK, Ching CTS, Sun TP, et al. Non-invasive and transdermal measurement of blood uric acid level in human by electroporation and reverse iontophoresis. Int J Nanomedicine. 2010;5:991-997.

15. Murthy SS, Kiran VS, Mathur SK, Murthy SN. Noninvasive transcutaneous sampling of glucose by electroporation. J Diabetes Sci Technol. 2008;2:250-254.

16. Merino V, Kalia YN, Guy RH. Transdermal therapy and diagnosis by iontophoresis. Trends Biotechnol. 1997;15:288-290.

International Journal of Nanomedicine

\section{Publish your work in this journal}

The International Journal of Nanomedicine is an international, peerreviewed journal focusing on the application of nanotechnology in diagnostics, therapeutics, and drug delivery systems throughout the biomedical field. This journal is indexed on PubMed Central, MedLine, CAS, SciSearch $\AA$, Current Contents $₫ /$ Clinical Medicine,
17. Neumann E, Schaefer-Ridder M, Wang Y, Hofschneider PH. Gene transfer into mouse lyoma cells by electroporation in high electric field. EMBO J. 1982;7:841-845.

18. Jadoul A, Bouwstra J, Preat V. Effect of iontophoresis and electroporation on the stratum corneum. Adv Drug Deliv Rev. 1999;35:89-105.

19. Tokumoto S, Higo N, Sugibayashi K. Effect of electroporation and $\mathrm{pH}$ on the iontophoresis transdermal delivery of human insulin. Int $J$ Pharm. 2006;326:13-19.

20. Loh KS, Lee YH, Musa A, Salmah AA, Zamri I. Use of $\mathrm{Fe}_{3} \mathrm{O}_{4}$ nanoparticles for enhancement of biosensor response to the herbicide 2,4dichlorophenoxyacetic acid. Sensors. 2008;8:5775-5791.

21. Zhang Y, Yuan R, Chai Y, Wang J, Zhong H. Amperometric biosensor for nitrite and hydrogen peroxide based on hemoglobin immobilized on gold nanoparticles/polythionine/platinum nanoparticles modified glassy carbon electrode. J Chem Technol Biotechnol. 2012;87:570-574.

22. Yao YL, Shiu KK. A mediator-free bienzyme amperometric biosensor based on horseradish peroxidase and glucose oxidase immobilized on carbon nanotube modified electrode. Electroanalysis. 2008;20:2090-2095.

23. Chen B, Ma M, Su X. An amperometric penicillin biosensor with enhanced sensitivity based on co-immobilization of carbon nanotubes, hematein, and beta-lactamase on glassy carbon electrode. Anal Chim Acta. 2010;674:89-95.

24. Zheng L, Li J, Xu J, et al. Improvement of amperometric glucose biosensor by the immobilization of $\mathrm{FcCD}$ inclusive complex and carbon nanotube. Analyst. 2010;135:1339-1344.

25. Liu J, Sun S, Liu C, Wei S. An amperometric glucose biosensor based on a screen-printed electrode and Os-complex mediator for flow injection analysis. Measurement. 2011;44:1878-1883.

26. Chen KJ, Chandrasekara Pillai K, Rick J, et al. Bimetallic PtM $(\mathrm{M}=\mathrm{Pd}$, Ir) nanoparticle decorated multi-walled carbon nanotube enzyme-free, mediator-less amperometric sensor for $\mathrm{H}_{2} \mathrm{O}_{2}$. Biosens Bioelectron. 2012;33:120-127.

27. Dai YQ, Shiu KK. Highly sensitive amperometric glucose biosensor based on glassy carbon electrode with copper/palladium coating. Electroanalysis. 2004;16:1806-1813.

28. Ching CTS, Chang KM, Hung YL, et al. An array configuration to increase the performance of a biosensor. Sens Actuators B Chem. 2013;178:465-472.

29. Ching CTS, Camilleri I, Connolly P. A low-cost, programmable device for versatile current delivery in iontophoresis applications. Sens Actuators B Chem. 2005; 106:534-540.

30. Ching CTS, Chih WY. Design and evaluation of an affordable and programmable mobile device, capable of delivering constant current and high voltage electric pulses of different waveforms for biomedical and clinical applications. Sens Actuators B Chem. 2014;194:361-370.

31. Zhao C, Wan L, Wang Q, Liu S, Jiao K. Highly sensitive and selective uric acid biosensor based on direct electron transfer of hemoglobin-encapsulated chitosan-modified glassy carbon electrode. Anal Sci. 2009;25:1013-1017.

32. Luo YC, Do JS, Liu CC. An amperometric uric acid biosensor based on modified Ir-C electrode. Biosens Bioelectron. 2006;22:482-488.

33. Chauhan N, Pundir CS. An amperometric uric acid biosensor based on multiwalled carbon nanotube-gold nanoparticle composite. Anal Biochem. 2011;413:97-103.

34. Zhang YQ, Shen WD, Gu RA, Zhu J, Xue RY. Amperometric biosensor for uric acid based on uricase-immobilized silk fibroin membrane. Anal Chim Acta. 1998;369:123-128.

Journal Citation Reports/Science Edition, EMBase, Scopus and the Elsevier Bibliographic databases. The manuscript management system is completely online and includes a very quick and fair peer-review system, which is all easy to use. Visit http://www.dovepress.com/ testimonials.php to read real quotes from published authors. 